\title{
Resultados inmediatos y alejados del switch arterial en pacientes con dextrotransposición de grandes arterias: experiencia de 20 años.
}

\author{
Pedro Becker, Matías Delgado , Patricia Frangini, Rodrigo González, Gonzalo Urcelay, Cristián Clavería \\ Francisco Garay, Pamela Zelada, Daniel Springmüller, Guillermo Lema, Jaime Cerda, Felipe Heusser.
}

\author{
División de Enfermedades Cardiovasculares; División de Pediatría; División de Anestesiología; Departamento de Salud Pública. \\ Facultad de Medicina; Pontificia Universidad Católica de Chile \\ a: Interno de $7^{\circ}$ año de Medicina
}

Objetivos: Comunicar los resultados de la operación de switch arterial en pacientes portadores de dextro Transposición de Grandes Arterias (D-TGA) y evaluar su evolución en el tiempo.

Pacientes y Método: Estudio retrospectivo de pacientes sometidos a switch arterial entre mayo de 1992 y noviembre de 2012. Se comparó período 1 (1992 a 2002) con período 2 (2003 a 2012). Se definió D-TGA simple aquella sin lesiones asociadas y D-TGA compleja aquella con asociación de comunicación interventricular o coartación aórtica.

Resultados: Un total de 108 pacientes componen la serie, 44 en el período 1 y 64 en el período 2 , sin diferencias demográficas y anatómicas entre ambos períodos. Setenta tenían D-TGA simple y 38 D-TGA compleja. La mortalidad operatoria en el período 1 fue $33 \%$ versus $8,4 \%$ en el período 2 ( $p<0.025$ ); hubo tendencia a mayor mortalidad en pacientes con anatomía coronaria compleja. Se observó una disminución del riesgo relativo de mortalidad de $68,7 \%$ en el período 2. El 28,7\% presentaron complicaciones postoperatorias, sin diferencias entre ambos períodos. Un paciente falleció en forma alejada. La mediana de seguimiento fue de 60 meses y la supervivencia de $84,25 \%$ a 10 y 20 años. Se realizaron 16 re intervenciones, principalmente plastías percutáneas de ramas pulmonares. La mayoría de los pacientes se mantenían asintomáticos. La mortalidad operatoria de los últimos 5 años fue $2,6 \%$.

Conclusiones: La mortalidad operatoria ha disminuido significativamente a lo largo de 20 años; persiste un mayor riesgo en ciertos patrones de anatomía coronaria. La supervivencia alejada y libre de reintervenciones es muy favorable. 


\section{Early and late results of the Switch operation for D-Transposition of the Great Vessels. Clinical experience in 108 patients operated on between 1992 and 2012}

Aim: to report the results of the arterial Switch operation in patients with D-transposition of the great vessels (D-TGA) and to evaluate their late course.

Patients and Methods: A retrospective review of the clinical data on patients consecutively operated on for D-TGA using the switch procedure. Results obtained in patients operated on between 1992 and 2002 (Period 1) were compared to those obtained in patients undergoing their operation between 2013 and 2012. Patients with D-TGA and no complications were compared to those who had interventricular septal defect or aortic coarctation associated their TGA.

Results: 44 patients belonged in Period 1 and 64 in Period 2 (total 108). Demographic and anatomical characteristics were similar in both periods. Simple DTGA was present in 70 patients and complex D-TGA in 38. Operative mortality was higher in Period 1 compared to Period 2 (33\% vs. 8.4\%, $\mathrm{p}<0.025)$. A higher, albeit not statiscally significant mortality was observed in patients with complex as opposed to simple D-TGA. A $68.7 \%$ relative reduction mortality risk was observed in Period 2. Complications developed in $28.7 \%$ of patients, with no difference between periods. Only 1 patient died during late follow up. Median follow up was 60 months and survival rate was $84.3 \%$ at 10 y 20 years. Sixteen patients required re-intervention, mainly to perform percutaneous plastic procedures on pulmonary artery branches. Most patients had an asymptomatic course during follow up. Surgical mortality for the last 5 years was $2.6 \%$.

Conclusion: surgical mortality for the arterial switch operation in patients with D-TGA has significantly decreased along a 20 year period. An increased surgical risk persists for patients with certain forms of coronary artery anomalies. Late survival free of reintervention was the rule in these patients.

Keywords: Transposition of the great vessels, D-TGA, Switch operation, clinical series.
Introducción: En 1976 el doctor Adib Jatene publicó los primeros casos exitosos de la operación de switch arterial para el tratamiento de los pacientes con dextrotransposición de las grandes arterias (D-TGA) ${ }^{1}$. Si bien esta cirugía inicialmente tuvo una alta mortalidad operatoria, rápidamente reemplazó a la operación de Senning $^{2}$ y a la operación de Mustard ${ }^{3}$ como tratamiento de elección para corregir esta grave cardiopatía congénita. Muchos grupos en el mundo experimentaron una curva de aprendizaje en una operación técnicamente más demandante que los procedimientos de Senning y Mustard, fundamentalmente por ser necesario transferir las arterias coronarias y por la necesidad de realizar esta intervención en el período neonatal, principalmente en aquellos pacientes sin comunicación interventricular (CIV) asociada $^{4}$.

En la Universidad Católica se inició el programa de cirugía de recién nacidos, lactantes y niños pequeños a partir del año 1989, incorporándose precozmente la operación de switch arterial (también conocida como operación de Jatene), inicialmente con resultados caracterizados por alta mortalidad operatoria, propios de un grupo aún con poca experiencia.

Al hacer una revisión en MEDLINE, no encontramos 
publicaciones nacionales con resultados inmediatos y alejados en pacientes con D-TGA sometidos a esta cirugía.

El objetivo del presente trabajo es comunicar los resultados inmediatos y alejados de la operación de switch arterial en pacientes portadores de D-TGA, investigar factores de riesgo, supervivencia, necesidad de re-intervenciones, capacidad funcional y evaluar la evolución de los resultados a lo largo de 20 años de experiencia.

Material y métodos: Se realizó una revisión retrospectiva de todos los pacientes consecutivos sometidos a switch arterial entre mayo de 1992 y noviembre de 2012; el cierre del estudio se hizo en septiembre de 2013 y se incluyeron pacientes operados hasta noviembre de 2012, de manera que el seguimiento mínimo fuera de al menos 10 meses. La información se obtuvo de la base de datos de cirugía cardíaca, fichas clínicas, fichas electrónicas, protocolos operatorios, contacto con los cardiólogos tratantes, contacto telefónico de pacientes y finalmente datos del Registro Civil e Identificación. Con el objetivo de evaluar evolución de los resultados en el tiempo, se dividió arbitrariamente el estudio en dos períodos: período 1(1992 a 2012) y período 2 (2003 a 2012).

El presente estudio contó con la aprobación del Comité de Ética de Investigación del Hospital Clínico de la Pontificia Universidad Católica de Chile.

Se definió como D-TGA simple aquella sin lesiones cardíacas mayores asociadas, excepto la presencia de una comunicación interauricular, un ductus arterioso persistente o estenosis subvalvular pulmonar leve. Se definió D-TGA compleja, aquella en que sí existe una lesión cardíaca mayor asociada, como CIV o coartación aórtica. La descripción de la anatomía coronaria se hizo basada fundamentalmente en la clasificación de Leiden ${ }^{5}$. Se excluyeron los pacientes sometidos a switch arterial portadores de doble salida de ventrículo derecho.

Para el análisis estadístico se utilizó el cálculo de riesgo relativo de mortalidad operatoria comparando ambos períodos, mediante modelo log binomial crudo y ajustado por variables confundentes. La comparación entre variables categóricas se hizo mediante test de Chi cuadrado y entre variables numéricas mediante test de Mann-Whitney; los cálculos de intervalos de confianza fueron del 95\%. Se utilizaron curvas de Kaplan Meier para supervivencia y supervivencia libres de re-intervención. Se utilizó el programa estadístico SPSS 15.0.

\section{Técnica Quirúrgica}

La operación se hizo en forma bastante estandarizada a lo largo de los años en cuanto a la forma de reconstrucción cardíaca: luego de tener al paciente conectado a circulación extracorpórea, la aorta es seccionada a nivel del punto medio entre el sitio de la cánula aórtica y la raíz aórtica; a continuación los botones coronarianos son tomados y movilizados a partir de su origen en la aorta. Posteriormente, el tronco de la arteria pulmonar es seccionado enfrentando el sitio de transección de la aorta. A continuación las coronarias son transferidas al tronco proximal de la arteria pulmonar seccionada, lo que ahora se denomina neoaorta proximal. Se realiza la maniobra de Lecompte, 6 en que la arteria pulmonar distal es transferida por delante de la aorta. Luego se realiza una anastomosis término-terminal entre la neoaorta proximal y la aorta ascendente distal. Se reconstruye la neopulmonar proximal desde donde fueron tomados los botones coronarianos, utilizando un parche de pericardio fijado en glutaraldehido, generalmente en la forma de un parche tipo pantalón. Finalmente, se hace una anastomosis término-terminal entre la neopulmonar proximal y la arteria pulmonar distal, la cual queda en posición anterior a la aorta. En todos los casos se cierra la comunicación interauricular y el ductus y según la anatomía se cierra además la CIV y se repara la coartación aórtica.

A lo largo de los años y según preferencia de los distintos cirujanos, hubo algunas variaciones en cuanto a la técnica de circulación extracorpórea y de transferencia de arterias coronarias.

Resultados: La serie está constituida por 108 pacientes; 44 operados en el período 1 y 64 en el período 2. El $65 \%$ correspondió a D-TGA simple y el $35 \%$ a DTGA compleja. Dentro de los 38 pacientes con D-TGA compleja, 24 tenían como lesión asociada una CIV, 10 tenían una CIV más una coartación aórtica y 4 tenían una CIV más estenosis subpulmonar considerada leva a moderada. En 7 de los pacientes con coartación aórtica se optó por reparar la coartación por toracotomía izquierda y algunos días después realizar el switch arterial con cierre de CIV; en 3 pacientes se hizo toda la reparación del switch arterial más la CIV y la coartación aórtica en un solo tiempo operatorio a través de una esternotomía media. Esta diferencia fue debida a preferencias del cirujano tratante.

Respecto de la anatomía coronaria, 70 pacientes eran 
portadores de una distribución del tipo Leiden 1, vale decir, del seno coronariano $\mathrm{N}^{\circ} 1$ emerge la coronaria izquierda que se bifurca en descendente anterior y circunfleja y del seno coronariano $\mathrm{N}^{\circ} 2$ nace la arteria coronaria derecha; 18 pacientes correspondían a Leiden tipo 2, en que del seno coronariano $\mathrm{N}^{\circ} 1$ nace exclusivamente la arteria descendente anterior y del seno coronariano $\mathrm{N}^{\circ} 2$ nacen la coronaria derecha y la arteria circunfleja. En 20 pacientes se observaron otras variantes, como se detalla en la Tabla 1.

\begin{tabular}{|c|c|}
\hline \multicolumn{2}{|c|}{$\begin{array}{c}\text { Tabla 1: Anatomía Coronaria en pacientes operados } \\
\text { por D-TGA mediante switch arterial }\end{array}$} \\
\hline Leiden 1 (1 DA, Cx; 2 ACD) & 70 \\
\hline Leiden 2 (1 DA; 2 ACD Cx) & 18 \\
\hline Leiden 3 (2 ACD, DA, Cx) & 0 \\
\hline Leiden 4 (1 ACD; 2 DA, Cx) & 1 \\
\hline Leiden 5 (1 DA, ACD; 2 Cx) & 7 \\
\hline Leiden 6 (2 ACD; 2 DA, Cx) & 5 \\
\hline ACI intramural & 2 \\
\hline DA intramural & 2 \\
\hline ACD intramural & 2 \\
\hline \multirow[t]{2}{*}{ Otro } & 1 \\
\hline & 108 \\
\hline \multicolumn{2}{|c|}{ Leyenda: ACI: arteria coronaria izquierda } \\
\hline \multicolumn{2}{|l|}{ DA: arteria descendente anterior } \\
\hline \multicolumn{2}{|l|}{ Cx: arteria circunfleja } \\
\hline ACD: arteria coronaria derech & \\
\hline
\end{tabular}

El 68\% de los pacientes fue sometido a una septostomía de Rashkind ${ }^{7}$ previo al switch arterial, en tanto que 32 pacientes no lo requirieron; esta distribución coincide bastante bien con la proporción de pacientes con y sin CIV asociada.

En la Tabla 2 se detallan las características demográficas,

\begin{tabular}{|l|l|l|l|}
\hline Tabla 2: Características de los pacientes según período de la intervención \\
\hline & Período 1 & Período 2 & p \\
\hline Edad (mediana) días & $8(6)$ & $7(8)$ & 0,766 \\
\hline Peso <3000 g & $9(21,4 \%)$ & $10(15,6 \%)$ & 0,451 \\
\hline Peso $\geq 3000 \mathrm{~g}$ & $33(78,6 \%)$ & $54(84,4 \%)$ & \\
\hline Peso (mediana), kg & $3.275(536)$ & $3.400(634)$ & 0,132 \\
\hline DTGA simple & $25(56,8 \%)$ & $45(70,3 \%)$ & 0,158 \\
\hline DTGA compleja & $19(43,2 \%)$ & $19(29,7 \%)$ & \\
\hline Leiden 1 & $24(54,5 \%)$ & $46(71,9 \%)$ & 0,012 \\
\hline Leiden 2 & $13(29,5 \%)$ & $5(7,8 \%)$ & \\
\hline Leiden Otra & $7(15,9 \%)$ & $13(20,3 \%)$ & \\
\hline Tpo. CEC (mediana) min. & $179(47)$ & $206(40)$ & 0,001 \\
\hline Tpo. Clampeo (mediana) min. & $92(18)$ & $98(34)$ & 0,351 \\
\hline Paro NO & $30(68,2 \%)$ & $52(81,3 \%)$ & 0,169 \\
\hline Paro SI & $14(31,8 \%)$ & $12(18,8 \%)$ & \\
\hline Tpo. Paro (mediana) min & $6(4)$ & $10(3)$ & 0,063 \\
\hline Cierre esternal dif. NO & $24(54,5 \%)$ & $20(31,3 \%)$ & 0,018 \\
\hline Cierre esternal dif. SI & $20(45,5 \%)$ & $44(68,8 \%)$ & \\
\hline
\end{tabular}

anatómicas y relativas a aspectos quirúrgicos en ambos períodos. No hubo diferencias en cuanto a edad, peso, tiempo de clampeo aórtico ni en cuanto a necesidad de paro circulatorio con hipotermia profunda. En el período 2 se aprecia una mayor proporción de pacientes con anatomía coronaria del tipo Leiden 1, hay un mayor tiempo total de circulación extracorpórea y una mayor proporción de pacientes con cierre esternal diferido. También se observó una tendencia a mayor proporción de D-TGA simple, pero no significativa.

Respecto de la técnica de perfusión y circulación extracorpórea, se observó una evolución en el tiempo existiendo inicialmente una mayor proporción de pacientes operados en hipotermia profunda y bajo flujo $(50 \mathrm{ml} / \mathrm{kg} /$ $\mathrm{min}$ ), asociado a un breve período de paro circulatorio para cierre de la comunicación interauricular (y en un caso para el cierre de la comunicación interventricular). Así, en el primer período 14 pacientes fueron operados bajo esta modalidad de hipotermia profunda y bajo flujo, contra 12 pacientes en el período 2. En ambos períodos los pacientes fueron operados mayoritariamente con hipotermia moderada, utilizando canulación de ambas venas cavas, aboliendo la necesidad de paro circulatorio.

Respecto de la técnica de transferencia de las coronarias, ésta ha variado principalmente en relación a preferencias de cada cirujano (Figura 1). Tanto para la coronaria del seno 1 como del seno 2 la técnica mayormente utilizada

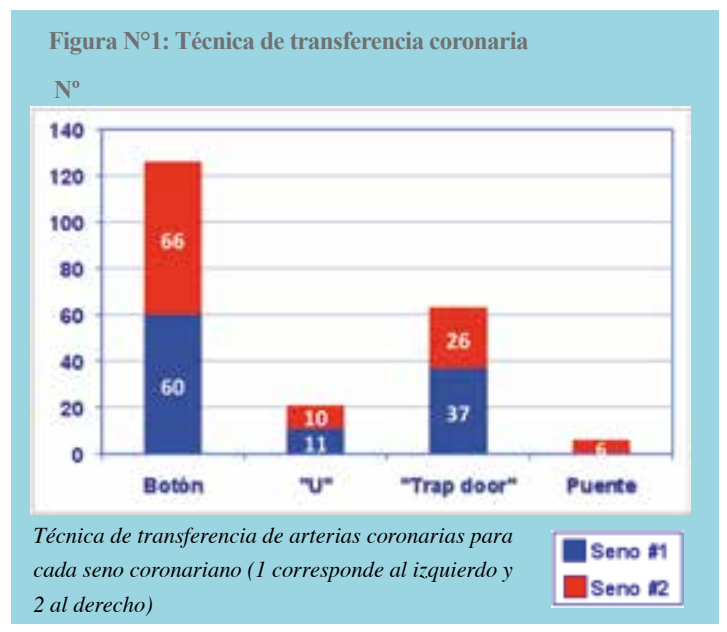

fue la de botón; la técnica de "Trap-door" fue la segunda modalidad más empleada, más frecuentemente para la transferencia de la coronaria del seno 2. En menor proporción se utilizó la técnica en U y finalmente en 6 
pacientes se empleó un puente aorto-coronario con interposición de carótida izquierda, para aquellos pacientes portadores de una anatomía coronaria del tipo Leiden 5 , con la arteria coronaria derecha naciendo desde la descendente anterior, principalmente, para evitar tensión en la coronaria derecha.

La mortalidad operatoria fue de 16 pacientes, 11 en el período $1(33 \%)$ y 5 en el período $2(8,4 \%)(\mathrm{p}<0,025)$. Dentro de las causas de mortalidad operatoria la principal fue la disfunción del ventrículo izquierdo, muy probablemente secundario a isquemia miocárdica. Hubo sólo 1 paciente que falleció en pabellón, secundario a isquemia miocárdica no corregible (Tabla 3). El riesgo relativo crudo de mor-

\begin{tabular}{|c|c|}
\hline & $\mathrm{N}$ \\
\hline Falla de ventrículo izquierdo & 5 \\
\hline Taponamiento cardíaco post retiro cables MCP & 1 \\
\hline Incapacidad salir de circulación extracorpórea & 1 \\
\hline Bradicardia extrema e hipotensión & 1 \\
\hline Disociación electro - mecánica & 1 \\
\hline Durante re-intervención por sangrado & 1 \\
\hline Durante re-intervención por sospecha mediastinitis & 1 \\
\hline Desconocida & 5 \\
\hline Total & 16 \\
\hline
\end{tabular}

talidad operatoria en el período 2 en relación al período 1 fue de 0,313 (IC 95\% 0,117- 0,887), lo cual significa una reducción del riesgo relativo de mortalidad del período 2 de $68,7 \%$. Al ajustar este riesgo relativo por anatomía de la D-TGA, anatomía coronaria y cierre esternal diferido, este riesgo relativo fue de 0,305 (IC 95\% 0,110 - 0,848) por lo que se mantiene una reducción significativa del riesgo relativo de mortalidad en el período 2 .

Al analizar la mortalidad operatoria en relación a la anatomía coronaria, no se encontraron diferencias significativas, si bien se observó una tendencia hacia una mayor mortalidad en el grupo con anatomía Leiden 4, con un solo caso operado que falleció y con la anatomía Leiden 6 , con 5 casos operados en el período, falleciendo 4 de ellos $(80 \%)$.

En 31 pacientes se produjo alguna complicaciones postoperatoria, 13 en el período 1 y 18 en el período 2 ; 16 pacientes presentaron una complicación y 15 pacientes presentaron dos o más, siendo las del tipo respiratoria e infecciosas las más frecuentes (Tabla 4). Dentro de las complicaciones respiratorias, las más frecuentes fueron el quilotórax y la parálisis diafragmática; dentro las

\begin{tabular}{|c|c|c|}
\hline & & $\mathrm{N}$ \\
\hline \multirow[t]{7}{*}{ Respiratoria } & & 17 \\
\hline & Quilotórax & 6 \\
\hline & Parálisis diafragmática & 5 \\
\hline & Derrame pleural & 4 \\
\hline & Pneumotórax & 3 \\
\hline & Hidrotórax & 2 \\
\hline & Atelectasia que requiere reconexión a VM & 2 \\
\hline \multirow[t]{6}{*}{ Infecciosa } & & 12 \\
\hline & Herida operatoria & 7 \\
\hline & Infección asociada a catéter & 2 \\
\hline & Fiebre de origen desconocido & 1 \\
\hline & Celulitis subclavia & 1 \\
\hline & Sepsis $2^{\circ}$ enterocolitis necrotizante & 1 \\
\hline \multirow[t]{5}{*}{ Vascular } & & 4 \\
\hline & Trombosis vena cava superior & 2 \\
\hline & Trombosis arterial extremidad inferior & 1 \\
\hline & Estenosis vena cava superior & 1 \\
\hline & Trombosis vena subclavia e iliaca & 1 \\
\hline \multirow[t]{4}{*}{ Neurológica } & & 3 \\
\hline & Sd. Convulsivo & 3 \\
\hline & Trombosis seno venoso & 1 \\
\hline & $\begin{array}{l}\text { Infarto cerebral parieto-occipital } \\
\text { con conversión hemorrágica }\end{array}$ & 1 \\
\hline \multirow[t]{3}{*}{ Digestivo } & & 3 \\
\hline & Enterocolitis necrotizante & 2 \\
\hline & Ascitis & 1 \\
\hline \multirow[t]{2}{*}{ Renal } & & 2 \\
\hline & Oliguria con requerimiento peritoneodiálisis & 2 \\
\hline \multirow[t]{2}{*}{ Hematológico } & & 3 \\
\hline & Sangrado que requiere reoperación & 3 \\
\hline $\begin{array}{l}\text { VM: ventilación me } \\
* 31 \text { pacientes tuvier } \\
\text { tuvieron una compl }\end{array}$ & $\begin{array}{l}\text { licaciones: } 13 \text { en el período } 1, \text { y } 18 \text { en el período } 2.16 \text { pacier } \\
15 \text { pacientes tuvieron } 2 \text { o más complicaciones }\end{array}$ & \\
\hline
\end{tabular}

infecciosas, lo fue la infección de herida operatoria, no existiendo diferencias significativas entre pacientes con y sin cierre esternal diferido. Hubo 2 casos de trombosis de vena cava superior, en que fue necesario realizar una trombectomía precoz y un caso de estenosis de vena cava superior que requirió de dilatación e instalación de un stent. Tres pacientes requirieron re-operación por sangrado postoperatorio y 2 requirieron peritoneodiálisis por insuficiencia renal aguda. Las complicaciones cardiovasculares relevantes fueron letales y por lo tanto están incluidas dentro de las causas de mortalidad operatoria. Prácticamente todos los pacientes desarrollaron algún período de bajo débito cardíaco, lo cual es habitual en esta cirugía. Sin embargo, esto fue una situación transitoria y en todos los pacientes preventivamente se utilizó asociación de drogas vasoactivas desde algunos minutos antes de la desconexión de circulación extracorpórea, manteniendo inótropos hasta después de la extubación del paciente en la de Unidad de Cuidados Intensivos. 
La mediana de seguimiento fue 60 meses (10 a 204) con un seguimiento completo global de $63,7 \%$. Respecto de la supervivencia, el seguimiento se consideró completo en un $100 \%$ por cuanto se utilizó como fuente de información el Registro Civil e Identificación. La mortalidad alejada correspondió a 1 paciente quien requirió angioplastía percutánea y luego quirúrgica por estenosis residual supravalvular aórtica y pulmonar; posterior a esta cirugía, el paciente presentó un paro cardíaco secundario a taponamiento cardíaco y evolucionó con encefalopatía hipóxico-isquémica como secuela; falleció 4 años más tarde por insuficiencia respiratoria.

En la Figura 2 se aprecia la curva de supervivencia global observando que la mayor atrición se produjo dentro del primer mes producto de la mortalidad operatoria, manteniéndose posteriormente una supervivencia estable a lo largo de estos 20 años de seguimiento $(84,25 \%)$. Se realizaron 33 re-intervenciones en 12 pacientes, siendo

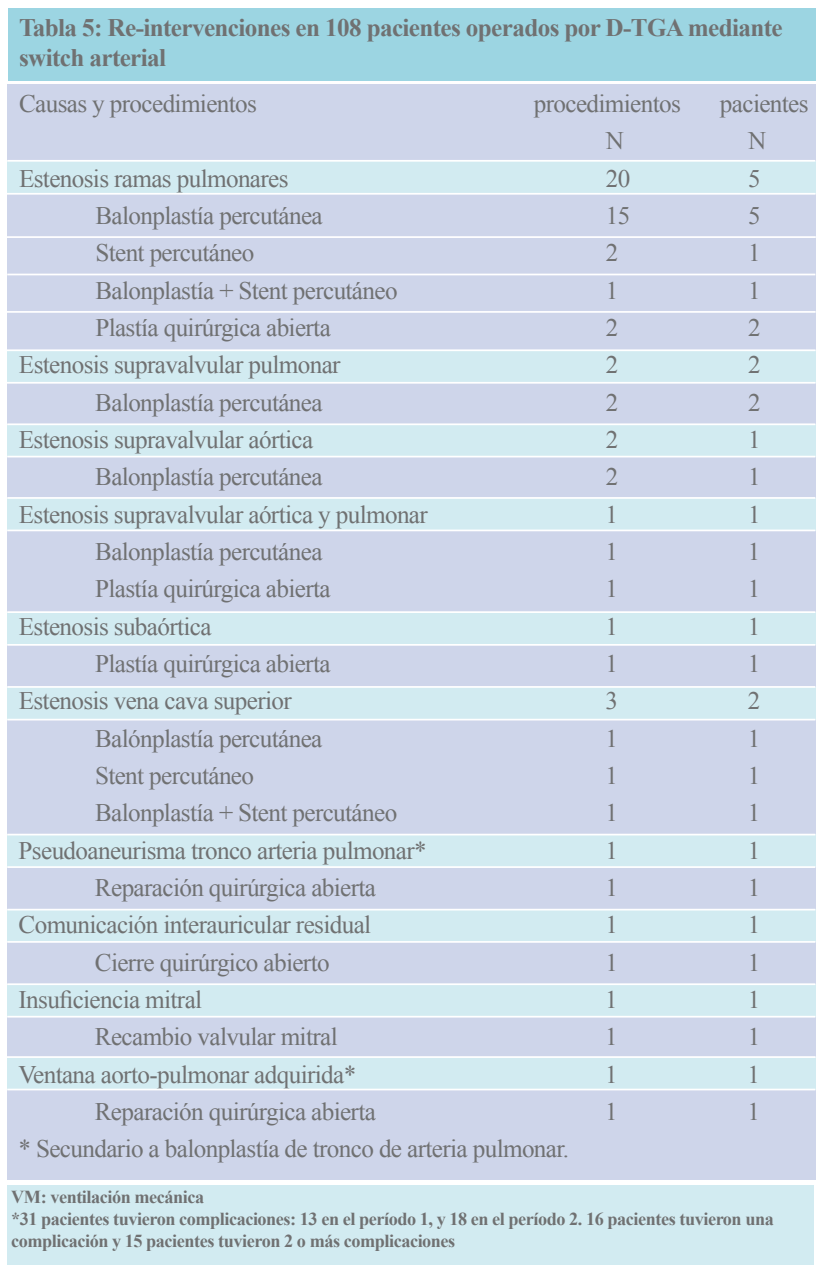

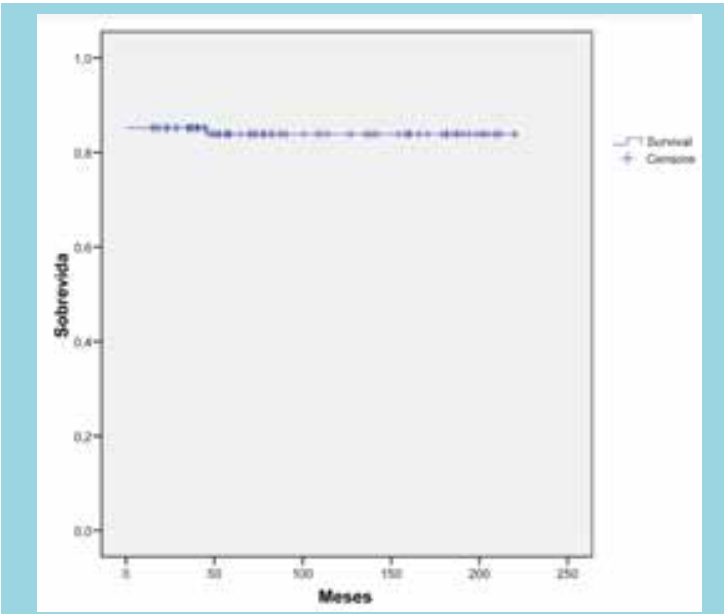

Figura 2: Curva de supervivencia actuarial en pacientes con D-TGA sometidos a switch arterial.

los procedimientos sobre estenosis de ramas pulmonares las más frecuentes (Tabla 5). Hubo dos pacientes que tuvieron complicaciones secundarias a procedimientos percutáneos para tratar estenosis de ramas pulmonares o estenosis supravalvular pulmonar: en uno de ellos se produjo un pseudoaneurisma del tronco de arteria pulmonar y en otro una ventana aorto-pulmonar; ambos pacientes fueron reparados quirúrgicamente en forma exitosa. Un paciente desarrolló insuficiencia mitral un año después de la cirugía, requiriendo reemplazo valvular mitral con prótesis mecánica. En la Figura 3 se aprecia la curva de supervivencia libre de re-intervenciones, observándose que la mayoría de éstas se producen dentro de los primeros 5 a 6 años de evolución postoperatoria. No hemos obser-

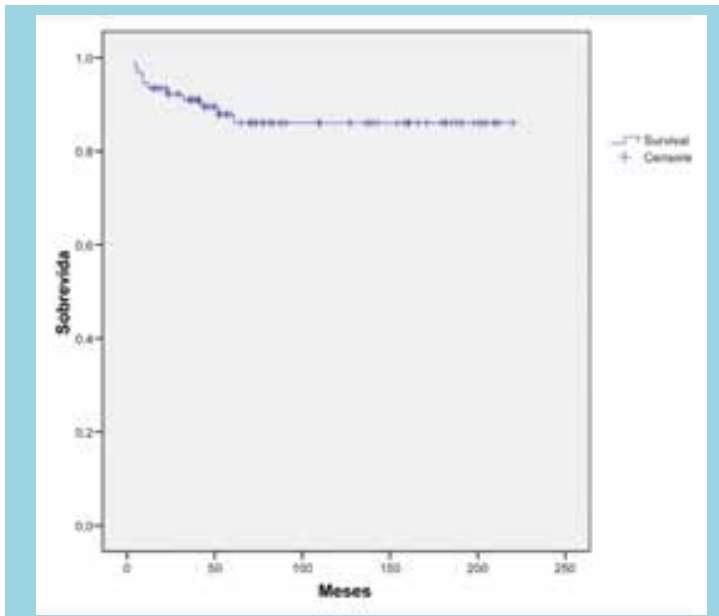

Figura 3: Curva de supervivencia libre de re-intervención en pacientes con D-TGA sometidos a switch arterial 
vado aún pacientes con oclusión de arterias coronarias o insuficiencia de la válvula neoaórtica que hayan requerido re-intervención. En la Figura 4 se aprecia la supervivencia libre de re-intervención comparando el período 1 con

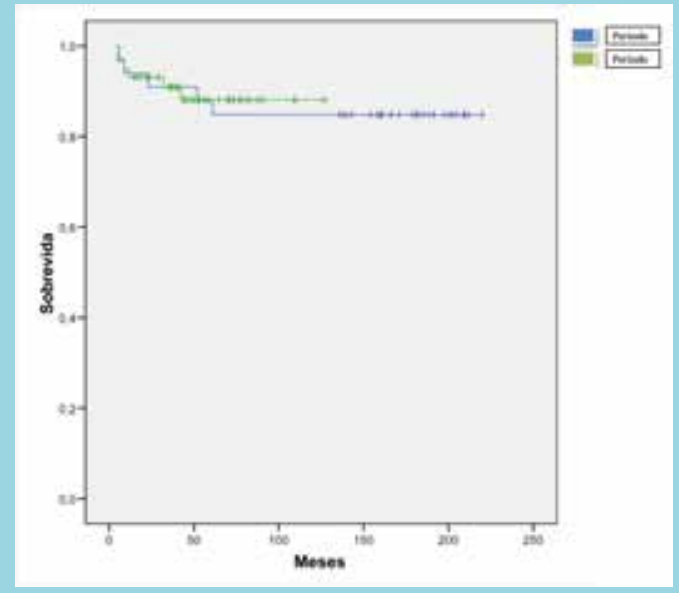

Figura 4: Curva de supervivencia libre de re-intervención, según período de la intervención

el 2, no apreciando diferencias. En 46 de los 91 supervivientes (50,5\%), se logró actualizar el estado funcional de los pacientes (Tabla 6). La mayoría de ellos se mantenían asintomáticos o con limitación leve de su capacidad física; así mismo, la mayoría asistían a jardín infantil o colegio

\begin{tabular}{|l|l|}
\hline $\begin{array}{c}\text { Tabla 6: Estado funcional de los pacientes operados por } \\
\text { D-TGA mediante switch arterial }\end{array}$ \\
\hline Clínica & N \\
\hline Asintomático (CF-I) & 30 \\
\hline Síntomático (CF-II a III) & 16 \\
\hline Comorbilidades CV & 5 \\
\hline Hipertensión arterial & 5 \\
\hline Medicamentos & 9 \\
\hline IECA & 3 \\
\hline B- bloqueador & 3 \\
\hline TACO* & 2 \\
\hline AAS & 2 \\
\hline Asistencia Jardín/Colegio & 34 \\
\hline Colegio Normal & 32 \\
\hline Colegio especial & 2 \\
\hline No asiste & 7 \\
\hline Atraso de curso & 4 \\
\hline $\begin{array}{l}\text { TACO: tratamiento anticoagulante oral, IECA: inhibidor enzima convertidora } \\
\text { de angiotensina, AAS: ácido acetil salicilico, }{ }^{*} \text { Uso por stent en vena cava superior } \\
\text { y prótesis mitral }\end{array}$ \\
\hline
\end{tabular}

normal. Cinco pacientes presentaban hipertensión arterial, 8 utilizaban algún medicamento, como inhibidores de la enzima convertidora de angiotensina o betabloqueadores y 2 requierían tratamiento anticoagulante oral crónico (por stent en vena cava superior y por prótesis mitral mecánica).

En la Figura 5 se grafica la evolución del número de operaciones por año y la mortalidad anual a lo largo de estos 20 años; se aprecia que en la medida que ha aumentado la experiencia del grupo, la mortalidad ha ido disminuyendo progresivamente, llegando a $2.6 \%$ en los últimos 5 años (2008 a 2012).

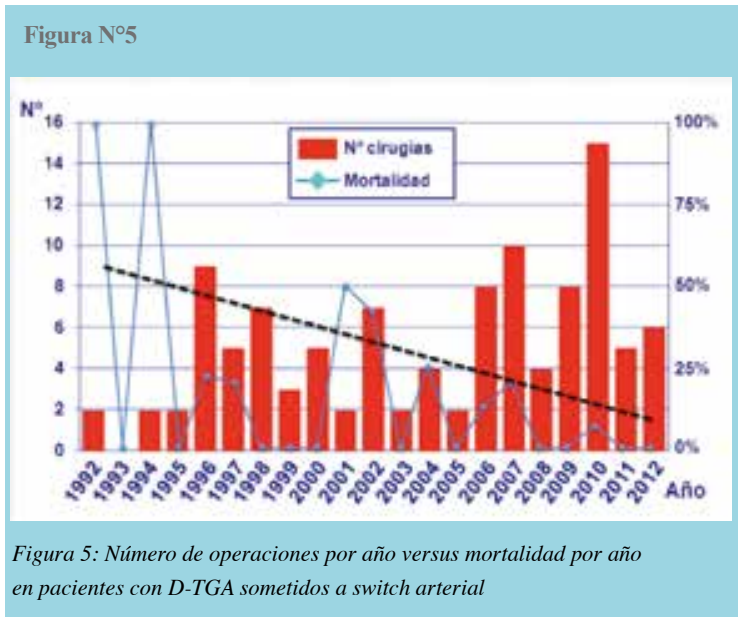

Discusión: La D-TGA es una cardiopatía cianótica grave en que existe concordancia aurículo-ventricular pero discordancia ventrículo-arterial, emergiendo la arteria aorta del ventrículo derecho y la arteria pulmonar del ventrículo izquierdo. Por lo tanto existe circulación en paralelo, por cuanto la sangre poco oxigenada del ventrículo izquierdo es enviada al cuerpo a través de la aorta y la sangre oxigenada es enviada a los pulmones a través de la arteria pulmonar. La prevalencia descrita es del orden de 0,24 por cada 1.000 nacidos vivos ${ }^{8}$. Considerando que Chile tiene una tasa anual de alrededor de 230.000 partos, se espera para nuestro país una incidencia de unos 50 casos nuevos cada año.

Esta enfermedad congénita fue inicialmente tratada quirúrgicamente mediante redistribución de flujos a nivel de las aurículas (switch auricular), procedimientos conocidos como la operación de Senning y la operación de Mus$\operatorname{tard}^{2,3}$. Esta cirugía era muy eficiente en cuanto a resolver la cianosis, ya que redirigía la sangre de la aurícula de- 
recha hacia el ventrículo izquierdo (el cual se mantenía conectado con la arteria pulmonar) y se redirigía el flujo de sangre desde la aurícula izquierda hacia el ventrículo derecho (el cual mantenía su conexión natural con la aorta). Sin embargo, esta técnica presentaba algunas dificultades en el mediano y largo plazo, fundamentalmente por la presencia de disfunción del nodo sinusal y la mantención del ventrículo derecho soportando la circulación sistémica, con progresivo deterioro de su función ${ }^{9,10}$. A partir del año 1975 el Dr. Adib Jatene desarrolló una nueva operación consistente en cambiar de posición los grandes vasos, dejando ahora la aorta conectada al ventrículo izquierdo y la arteria pulmonar al ventrículo derecho; la primera experiencia fue publicada el año $1976^{1}$, y a partir de la década de los 80 se consolidó como el tratamiento quirúrgico de elección en pacientes con DTGA. Su principal atractivo radicó en dejar al ventrículo izquierdo soportando la circulación sistémica en lugar del ventrículo derecho. Pero se trataba de una operación técnicamente más compleja y demandante por lo que la mortalidad operatoria inicial fue muy elevada, de hasta incluso $100 \%$ en algunas instituciones ${ }^{4}$. Con el tiempo, gracias al perfeccionamiento de la técnica operatoria y de los cuidados postoperatorios, la mortalidad operatoria del switch arterial comenzó a disminuir, existiendo en la actualidad muchos centros que exhiben mortalidad muy por debajo del $10 \%{ }^{11,12,13}$.

Las primeras operaciones de switch arterial fueron realizadas en lactantes menores que tenían CIV asociada. Un avance importante fue la incorporación de esta operación al período neonatal, demostrándose que el ventrículo izquierdo era capaz de adaptarse a la mayor post-carga luego del switch arterial dentro de las primeras semanas de vida, en que existe la posibilidad de desarrollar hiperplasia miocárdica ${ }^{14}$. Es así como desde la década del 80 se consolidó el concepto de reparar esta cardiopatía mediante switch arterial en el período neonata ${ }^{15}$. De esta forma, esta operación se transformó en un ícono de la cirugía cardíaca compleja en recién nacidos y de alguna manera se constituyó en un indicador de la calidad de los distintos grupos cardioquirúrgicos en el mundo ${ }^{16}$.

En nuestra experiencia inicial, la mortalidad operatoria fue alta, similar a la que tuvo el propio Jatene en sus inicios, sin embargo, en la medida que el volumen de operaciones aumentó, adquiriendo mayor experiencia quirúrgica, en perfusión, en anestesia y cuidados intensivos neonatales, la mortalidad comenzó a disminuir progresi- vamente. De esta forma apreciamos que el riesgo relativo de mortalidad disminuyó significativamente en el período 2 comparado con el período 1, independientemente del tipo de D-TGA (simple versus compleja), de la anatomía coronaria y del cierre esternal diferido. Previamente, la presencia de CIV, otras malformaciones asociadas y ciertos patrones de anatomía coronaria, han sido descritos como factores de mayor riesgo ${ }^{17,18}$. En nuestra experiencia, estos factores no alcanzaron significancia, lo que podría deberse a falta de potencia estadística, sin embargo, se observó una tendencia a mayor mortalidad en patrones coronarios con gran parte de la masa miocárdica izquierda dependiente de coronarias que pasan por detrás de la arteria pulmonar o con un trayecto inter-arterial (Leiden 4 y 5$)^{5}$. No se observó mortalidad en pacientes con trayectos intramurales de coronarias. Respecto del cierre esternal diferido, no observamos mayor mortalidad, a diferencia de otras series ${ }^{13}$, lo que puede deberse al hecho de que esta maniobra ha sido utilizada con cierta liberalidad en nuestro grupo y también porque puede tener un efecto protector. Puede destacarse también, que la mayor mortalidad operatoria correspondió al grupo con D-TGA compleja del período 1 (31.7\%) y la menor se observó en aquellos con D-TGA simple del período $2(4,4 \%)$.

Respecto de la forma de transferir las arterias coronarias, en estos 20 años se utilizaron diferentes técnicas, principalmente, por preferencias de cada cirujano. Rudra et al, ${ }^{13}$ en un estudio de 25 años de experiencia, observó que el grupo en que se utilizó la técnica en U tuvo la menor tasa de oclusiones coronarias; nosotros no hemos investigado dirigidamente la permeabilidad coronaria, por lo que no pudimos hacer esa comparación; sin embargo no han habido eventos clínicos ni mortalidad alejada atribuibles a mala perfusión coronaria.

Las causas de muerte postoperatoria fueron principalmente la falla ventricular izquierda, que fueron de ocurrencia precoz, y aquellas de causa desconocida que ocurrieron en 5 pacientes; perturba no conocer o saber interpretar la causa de mortalidad, lo cual puede observarse en unidades de cuidados intensivos aún poco experimentadas en manejar pacientes de esta naturaleza. Es posible que esta sea la explicación ya que estos casos se concentraron en los primeros años de nuestra experiencia. Probablemente hubo un porcentaje importante de esos pacientes que fallecieron también por disfunción ventricular isquémica, aunque podría también explicarse por múltiples factores deri- 
vados de menor experiencia del grupo.

Un $13 \%$ de los sobrevivientes a la cirugía requirió alguna re-intervención, lo cual es comparable a otras experiencias $^{13}$. Tal como muestra la experiencia mundial, la mayoría de las re-intervenciones se realizaron sobre las arterias pulmonares, que al quedar por delante de la aorta luego de la maniobra de Lecompte, pueden quedar algo tensionadas. En este sentido, es un detalle técnico relevante realizar una adecuada movilización de las ramas pulmonares hasta ambos hilios, además de usar un parche de pericardio en pantalón bastante generoso, para también minimizar la incidencia de estenosis supravalvular pulmonar ${ }^{11}$. Esta modalidad de reconstruir la arteria pulmonar fue adoptada desde el inicio en nuestro grupo, lo que puede explicar que no haya diferencias en ambos períodos en cuanto a frecuencia de re-intervenciones.

La supervivencia actuarial es similar a la reportada internacionalmente, destacando que la principal atrición se produce dentro del primer mes postoperatorio y luego la supervivencia se mantiene muy estable, al menos por los primeros 20 años en nuestra experiencia ${ }^{11,12,13}$. Un paciente falleció alejadamente: si bien fue de causa respiratoria, correspondía a un paciente con secuelas neurológicas debidas a un paro cardíaco durante una re-intervención por complicaciones alejadas postoperatorias.

El estado funcional de nuestros pacientes se logró determinar solo en el 50,5\% de la serie, lo que se explica porque una proporción importante de pacientes operados en nuestra Institución provienen de regiones a través del sistema Auge, implementado a partir de 2005. No fue factible contactar directamente a todos los pacientes, pero la información recabada a través de sus cardiólogos tratantes nos permitió tener una idea razonable de su estado clínico. Así mismo, podemos considerar que nuestro sistema de atención es relativamente "cerrado", por cuanto si un paciente tiene alguna complicación relevante debiera ser referido a nuestra institución. Este hecho también permite tener un razonable grado de certeza de que los pacientes con alguna complicación alejada mayor o necesidad de re-intervención no debieran escapar a nuestro conocimiento. Complementariamente realizamos una revisión de pacientes adultos re-intervenidos por secuelas de switch arterial en el Instituto Nacional del Tórax (que es el centro de referencia nacional para adultos con cardiopatías congénitas), no encontrando pacientes re-intervenidos en ese Instituto que hubiesen sido previamente operados por nosotros.

Constituye aún un capítulo abierto conocer cuál será la evolución en el real largo plazo de estos pacientes, por cuanto estos sobrevivientes son aún adolescentes. Incluso en los grupos con experiencias más antiguas que la nuestra, los pacientes que han sobrevivido son aún adultos jóvenes ${ }^{13}$.

Finalmente, como se grafica en la Figura 5, en la medida que han transcurrido los años, y ha aumentado la experiencia acumulada en cuanto al número de casos, la mortalidad operatoria ha ido disminuyendo sostenidamente, alcanzando 2,6\% en los últimos 5 años lo que corresponde a un sólo paciente fallecido en este último período.

Las limitaciones del presente trabajo corresponden a su carácter retrospectivo, por lo cual parte de la información puede ser incompleta o inexacta y esto también afecta la calidad del seguimiento. Otra limitación es que no hemos establecido un protocolo formal de seguimiento de potenciales complicaciones alejadas, como ser la presencia de oclusión de arterias coronarias, fenómenos isquémicos y el desarrollo de insuficiencia en la válvula neoaórtica o dilatación de la raíz de la neoaorta. Si bien nuestro sistema de atención debiera captar estos pacientes por las características antes mencionadas, no es posible tener certeza de su real prevalencia.

En conclusión, la mortalidad operatoria ha disminuido significativamente, siendo de 2,6\% en los últimos 5 años. Se observó una tendencia a mayor mortalidad en ciertos patrones de anatomía coronaria. La mayoría de los pacientes no presenta limitaciones físicas, está libre de uso de medicamentos y realiza vida normal. La supervivencia alejada y libre de re-intervenciones es muy favorable hasta 20 años de seguimiento y comparable a lo reportado internacionalmente. 


\section{Referencias:}

1. JATENE A, FONTES V, PAULISTA P, SOUZA L, NEGER F, GALANTIER M, et al. Anatomic correction of transposition of the great vessels. J Thorac Cardiovasc Surg 1976;72:364-9.

2. SENNING A, SWEDEN S. Surgical correction of transposition of the great vessels. Surgery 1959;45;966-80.

3. MUSTARD W. Successful two-stage correction of transposition of the great vessels. Surgery 1964;55:469-72.

4. KIRKLIN J, BLACKSTONE E, TCHERVENKOV CH, CASTANEDA A, AND THE CONGENITAL HEART SURGEONS SOCIETY. Clinical outcomes after the arterial switch operation for transposition. Circulation 1992;86:1501-15.

5. JONAS R. Comprehensive Surgical Management of Congenital Heart Disease. Oxford University Press Inc, 2004; $1 ; 256-78$.

6. LECOMPTE Y, ZANNINI L, HAZAN E, JARREAU M, BEX J, VIET-TU T, et al. Anatomic correction of transposition of the great arteries. J Thorac Cardiovasc Surg 1981;82:629-31.

7. RASHIND W, MILLER W. Creation of an atrial septal defect without thoracotomy. A palliative approach to complete transposition of the great arteries. JAMA 1966;196:173-4.

8. KEANE J, LOCK J, FYLER D. NADAS' Pediatric Cardioloogy. Editorial Saunders Elsevier. 2006; 2: 645-62.

9. BYRUM C, BOVE E, SONDHEIMER H, KAVEY R, BLACKMAN M. Hemodynamic and electrophysiologic results of the Senning procedure for transposition of the great arteries. Am J Cardiol 1986;58:138-42.

10. GILLETTE P, KUGLER J, GARSON A, GUTGESELL H, DUFF D, McNAMARA D. Mechanisms of cardiac arrhythmias after the Mustard operation for transposition of the great arteries. Am J Cardiol 1980;45:1225-30.

11. HUTTER P, KREB D, MANTEL S, HITCHCOCK J, MEIJBOOM E, BENNINK B. Twenty-five years' experience with the arterial switch operation. J Thoracic Cardiovasc Surg 2002;124:790-7.

12. SARRIS G, CHATZIS A, GIANNOPOULOS N, KIRVASSILIS G, BERGGREN H, HAZEKAMP M, et al. The arterial switch operation in Europe for transposition of the great arteries: A multi-institutional study from the European Congenital Heart Surgeons Association. J Thoracic Cardiovasc Surg 2006; 132: 633-9.

13. RUDRA H, MAVROUDIS C, BACKER C, KAUSHAL S, RUSSELL H, STEWART R, et al. The arterial switch operation: 25-year experience with 258 patients. Ann Thorac Surg 2011; 92: 1742-6.

14. DI DONATO R, FIJII A, JONAS R, CASTAÑEDA A. Age-dependent ventricular response to pressure overload. Considerations for the arterial switch operation. J Thorac Cardiovasc Surg 1992; 104: 713-22.

15. NORWOOD W, DOBELL A, FREED M, KIRKLIN J, BLACKSTONE E, AND THE CONGENITAL HEART SURGEONS SOCIETY. Intermediate results of the arterial switch repair. A 20-institution study. J Thorac Cardiovasc Surg 1988; 96: 854-63.

16. DE LEVAL M, Lessons from the arterial switch operation. Lancet 2001;357:1814

17. WERNOVSKY G, MAYER J, JONAS R, HANLEY F, BLACKSTONE E, KIRKLIN J, et al. Factors influencing early and late outcome of the arterial switch operation for transposition of the great arteries. J Thorac Cardiovasc Surg 1995; 109: 289-302.

18. MAYER J, SANDERS S, JONAS R, CASTAÑEDA A, WERNOVSKY G. Coronary artery pattern and outcome of arterial switch operation for transposition of the great arteries. Circulation 1990;82: IV-139 - IV-145. 